\title{
Virtual textile composites software WiseTex: Integration with micro-mechanical, permeability and structural analysis
}

\author{
Ignaas Verpoest *, Stepan V. Lomov \\ Department of Metallurgy and Materials Engineering, Katholieke Universiteit Leuven, Kasteelpark Arenberg 44, B-3001 Leuven, Belgium
}

Received 6 May 2005; accepted 25 May 2005

Available online 26 July 2005

\begin{abstract}
The internal geometry of textile reinforcements is an important factor of the reinforcement performance during composite manufacturing and in the service life of the composite material. When a 3D-shaped composite part is concerned, the reinforcement is locally deformed (compressed, stretched and sheared), and any model describing the internal geometry of the reinforcement should account for this deformation. The software package WiseTex implements a generalised description of internal structure of textile reinforcements on the unit cell level, integrated with mechanical models of the relaxed and deformed state of 2D- and $3 \mathrm{D}$-woven, two- and three-axial braided, weft-knitted and non-crimp warp-knit stitched fabrics and laminates. It is integrated with modelling of resin flow, micro-mechanical calculations of properties of textile based composites and micro-macro analysis of composite parts, finite element models and virtual reality software. The paper describes this family of models, which use a unified description of the geometry of the reinforcement unit cell.
\end{abstract}

(C) 2005 Elsevier Ltd. All rights reserved.

Keywords: Textile composites; Modelling; Internal structure; Deformability; Permeability; Micro-mechanics; Finite elements

\section{Introduction}

The internal geometry of a textile reinforcement is an important factor of the reinforcement performance during composite manufacturing and in service life of the composite material. For the former, impregnation of the reinforcement is governed by its porosity (size, distribution and connectivity of pores). For the latter, load transfer from the matrix to the reinforcement is governed by the fibre orientation, which plays a paramount role in the composite stiffness; stress-strain concentration loci, determining the composite strength, are correlated with the resin rich zones and fibre/matrix interfaces, distributed in the composite volume in accordance with the reinforcement geometry. When a 3D

\footnotetext{
* Corresponding author. Tel.: +3216321210; fax: +3216321990 .

E-mail addresses: ignass.verpoest@mtm.kuleuven.ac.be (I. Verpoest), stepan.lomov@mtm.kuleuven.be (S.V. Lomov).
}

shaped composite part is concerned, the reinforcement is locally deformed (compressed, stretched and sheared), and the geometrical model should account for this deformation.

A generalised description of the internal structure of a textile reinforcement has been developed in K.U.Leuven. It is the final result of a development that started in the mid 1990s, with the work of Vandeurzen et al. [1,2] on woven fabric composites and Gommers et al. [3] on knitted and woven fabric composites. Both authors based the description of the internal geometry of the textile on empirical data on the yarn orientation, obtained by optical or electron-microscopical observations on dry textiles and on cross-sections of textile composites. For woven fabrics, the out-of-plane orientations of the warp and weft yarns at their cross-over points, were taken into account in an approximate way, by representing the curved yarns by a set of straight yarn segments. For knitted fabrics, a similar approach was used, but 
also the in-plane curvature of the knitted loops had to be represented.

These geometrical descriptions were then used to calculate the homogenised mechanical properties of the textile based composites. Vandeurzen [1,2] used a multilevel homogenisation method, also called the cell method, which was later on generalised by Prodromou [4]. Gommers [3] used a multi-inclusion Eshelby-type approach, in which each yarn segment is considered to be an inclusion embedded in a medium that has either the matrix properties (Mori-Tanaka method) or the already homogenised composite properties (self-consisting method). Predictions of homogenised stiffness properties were quite accurate for the knits and yarns considered. Some attempts were made to predict damage development, because both methods allow to calculate, be it in an approximate way, the stresses or strains at the lowest level (impregnated yarns and matrix), and hence to predict at which applied stress or strain level the local stresses or strain reach a critical value.

The major drawback of these early models was their dependency on empirically obtained descriptions of the internal geometry of the textile. This means that the textile first has to be produced, and then measured, and that no predictions on the behaviour of yet non-existing textile composites can be made. In this way, the value of these 'predictive' models is rather limited. A big step forward could be achieved when collaboration was started with S.V. Lomov, who developed a model for the internal geometry of 2D- and 3D-weaves (the CETKA-model [5-11]), based on a minimum number of topological data (weave style, inter-yarn distance) and yarn mechanical properties. The model is a mechanical model, as it applies a yarn deformation energy minimisation algorithm to predict the internal geometry of any 2D- and 3D-weave. Connecting this approach to the cell- or inclusion models of Gommers and Vandeurzen resulted a more versatile way to calculate the homogenised properties of textile based composites [12-15].

Since the year 1999, this approach has been systematically followed, extending the types of textiles to 2Dand 3D-woven [14-17], two- and three-axial braided [18], weft-knitted [19] and non-crimp warp-knit stitched
[20] fabrics and laminates [21]. The mechanical models not only generate the internal geometry of relaxed textiles, but also of textiles deformed in tension, compression and shear. The models are implemented in the software package WiseTex.

A further step was the full integration of the geometrical models with other predictive models relevant for composites processing and performance prediction. Permeability tensors can be predicted by modelling of resin flow through the reinforcement $[22,23]$; the homogenised mechanical properties and the local stresses and strains can be generated by micro-mechanical calculations of properties of the composite [14,15], and can then be further linked to a micro-macro analysis of the composite parts $[24,25]$, finite element models [26-30] and virtual reality software [31] (Table 1 and Fig. 12). All these models use a unified description of the geometry of the reinforcement unit cell.

As is evidenced by Table 1, the WiseTex family of software is now the product of an intensive and widespread collaboration between different universities and research centres worldwide: University of Osaka, Japan (Prof. Zako, H. Nakai, S. Hirosawa), Politecnico de Milano, Italy (Prof. Poggi, Prof. Carvelli), Technical University of Liberec, Czech Republic (T. Mikolanda), University of Zaragoza, Spain (E. Bernal), ONERA, France (F. Boust, B. Laine) and ESI, France (P. de Luca, L. Dufort). Further contributions to the model and software development, reported in this paper, were made by a group of researchers in our own department (J-F. Delerue, S. Kondratiev, G. Huysmans, F. Tumer, B. van den Broucke) and in the Department of Computer Science of our university (Prof. D. Roose, T. Peeters, B. Verleye).

The present paper summarises these developments, with an aim to facilitate its future use of the unified textile models by other researchers.

\section{Unified description of geometry of unit cell and its translation to FE models}

The geometrical and mechanical model of textiles, implemented in the software package WiseTex, provides

Table 1

WiseTex family of software

\begin{tabular}{|c|c|c|}
\hline & Purpose & Developed by \\
\hline WiseTex & $\begin{array}{l}\text { Internal geometry of textiles in relaxed state; } \\
\text { compression, tension and shear of the textile }\end{array}$ & Lomov, Verpoest, K.U.Leuven \\
\hline LamTex & Internal geometry of textile laminates & Lomov, Peeters, K.U.Leuven \\
\hline FETex & Convertor of WiseTex data into FE description & $\begin{array}{l}\text { Lomov, Kondratiev, Bernal, K.U.Leuven; } \\
\text { Poggi, Carvelli, Politechnico di Milano }\end{array}$ \\
\hline MeshTex & Convertor of WiseTex data into FE description (SACOM) & Zako, Nakai, Hirosawa, Osaka University \\
\hline TexComp & Stiffness properties of a textile composite, inclusion model & Huysmans, Prodromou, Lomov, Verpoest, K.U.Leuven \\
\hline FlowTex & Permeability of textile, lattice Boltzmann/finite difference & Belov, Peeters, Roose, Verleye K.U.Leuven \\
\hline Celper & Permeability of textile, finite elements, voxel discretisation & Laine, Boust, ONERA \\
\hline VRTex & Virtual reality of textiles on meso-level & Mikolanda, T.U.Liberec \\
\hline
\end{tabular}


a full description of the internal geometry of a fabric: 2D- and 3D-woven, two- and three-axial braided, knitted, multi-axial multi-ply stitched (non-crimp fabric). Input data include: (1) Yarn properties: geometry of the cross-section, compression, bending, frictional and tensile behaviour, fibrous content; (2) Yarn interlacing pattern; (3) Yarn spacing within the fabric repeat. Models for compression, bi- and uni-axial tension and shear of the fabric are also based on an energy balance and calculate the internal structure of the deformed fabric, as well as load-deformation relation.

As an example of application of principle of minimum energy, consider the model of internal structure of 2D- or 3D-woven fabric (Fig. 1). A weave pattern (for one- and multi-layered fabrics) is coded with matrix coding [7,16], Fig. 1(a). It allows separation of the crimped shape of the warp and weft yarns into elementary bent intervals (), representing sections of the yarn between interlacing sites. The shape of the yarn on an elementary interval is described using a parameterised function $z(x ; h / p)$, where $z$ and $x$ are coordinates of the yarn middle line, $h$ is the crimp height and $p$ is the distance between the interval ends (spacing of the yarns). The shape $z(x ; h / p)$ for a given relative crimp height $h / p$ is computed using the principle of minimum of bending energy of the yarn on the interval and has a form

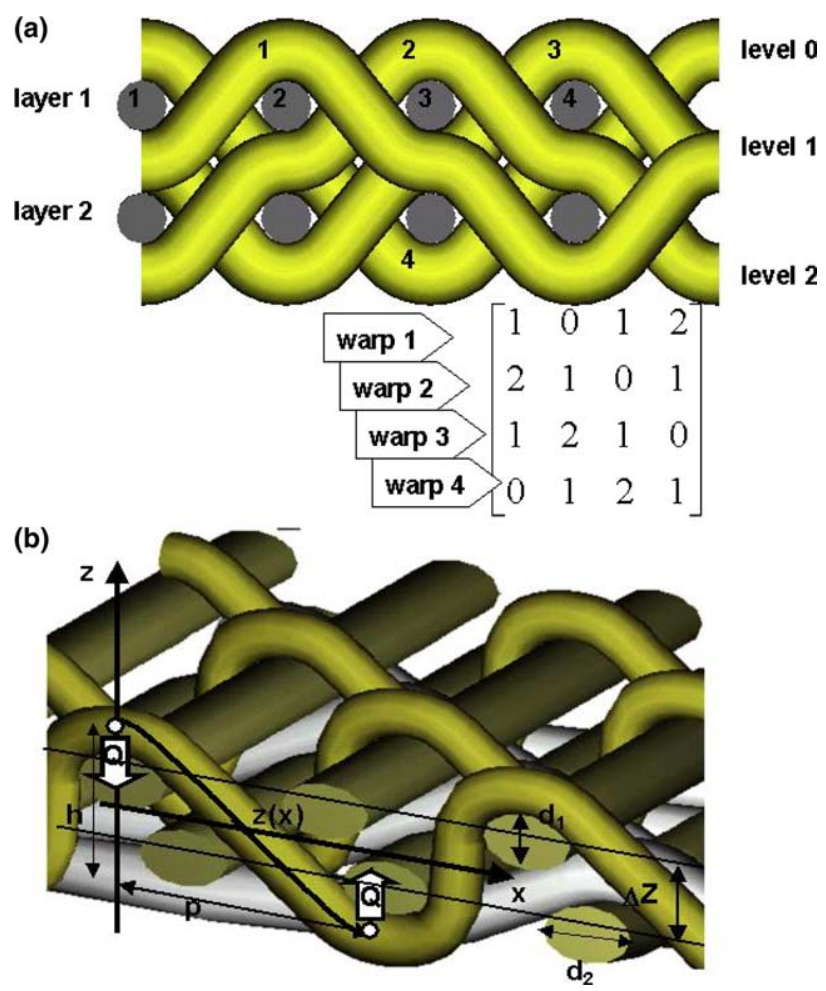

Fig. 1. Model of internal structure of woven fabric: (a) coding of the weave; (b) description of the yarn paths.

$$
\begin{aligned}
z(x)= & h\left[1 / 2-3(x / p)^{2}+4(x / p)^{3}\right. \\
& \left.+A(h / p)(x / p)^{2}((x / p)-1)^{2}((x / p)-1 / 2)\right],
\end{aligned}
$$

where the first term is a spline function, corresponding to the solution of the linearised minimum energy problem, and the second term represents a correction for a non-linear formulation. The function $A(h / p)$ is calculated from the solution of the minimum energy problem and is tabulated.

With this function known, the characteristic function $F$ of the crimp interval is computed, representing the bending energy of the yarn,

$w=\frac{1}{2} \int_{0}^{p} B(\kappa) \frac{\left(z^{\prime \prime}\right)^{2}}{\left(1+\left(z^{\prime}\right)^{2}\right)^{5 / 2}} \mathrm{~d} x=\frac{B(\bar{\kappa})}{p} F(h / p)$,

where $B(\kappa)$ is the (measured experimentally) bending rigidity of the yarn, which depends (non-lineary) on the local curvature $\kappa(x)$, or, after the integration, on an average curvature over the interval. Function $F(h / p)$ is tabulated. With the function $F$ known, the transversal forces acting on the interval ends can be estimated as

$Q=\frac{2 w}{h}=\frac{2 B(\bar{\kappa})}{p^{2}} \frac{p}{h} F(h / p)$.

Warp and weft yarns in the relaxed fabric are compressed by the transversal forces $Q$ according to experimental diagrams, measured on "virgin" yarns

$d_{1}=d_{10} \eta_{1}(Q), \quad d_{2}=d_{20} \eta_{2}(Q)$

where subscript " 0 " refer to the uncompressed state of the yarn, $d_{1}$ and $d_{2}$ are dimensions of the yarn cross-section (Fig. 1(b)). These dimensions and crimp heights of the yarns are interconnected

$h^{\mathrm{Wa}}=\Delta Z+\left(d^{\mathrm{Wa}}+d^{\mathrm{We}}\right)-\left(h_{1}^{\mathrm{Wa}}+h_{2}^{\mathrm{We}}\right) / 2$,

where superscripts refer to the warp and weft yarns, subscripts " 1 " and " 2 " refer to two weft yarns in different layers, $\Delta Z$ is the distance between fabric layers (Fig. 1(b)). With crimp heights of weft yarns given, these equations provide a closed system of non-linear equations for calculation of the transversal forces $Q$ and yarn dimensions $d_{1}$ and $d_{2}$. The weft crimp heights are found using the principle of minimum bending energy of the yarns inside the unit cell

$W_{\sum}=\sum_{i=1}^{N_{\mathrm{Wa}}} \sum_{k=1}^{K_{i}^{\mathrm{Wa}}} w_{i k}^{\mathrm{Wa}}+\sum_{j=1}^{N_{\mathrm{We}}} \sum_{k=1}^{K_{j}^{\mathrm{We}}} w_{j k}^{\mathrm{We}} \rightarrow \min$,

where subscripts $i, j$ refer to different warp and weft yarns, $k$ - to the elementary crimp interval of the warp/weft yarn. The minimum problem is solved for the weft crimp heights, all other parameters defined inside the minimisation algorithm via solution of the system for the given current crimp heights. It takes about $10 \mathrm{~s}$ on a $1 \mathrm{MHz}$ PC to compute parameters for 
a $3 \mathrm{D}$ fabric with 20 yarns in the repeat and about 0.5 $\mathrm{s}$ - for a plain weave fabric.

The description of the internal geometry is unified both for deformed and undeformed fabrics. This section explains this description and algorithms of its conversion into FE models using an integrated data transfer road map (Fig. 2). The reader is referred to the papers cited above for full formulation and experimental validation of the WiseTex models.

\subsection{Yarns}

\subsubsection{Description of the yarn geometry in the textile modelling software}

Consider a fabric consisting of yarns only (fibrous plies in NCF are discussed below). Fig. 3 illustrates the description of the spatial configuration of the yarns. The midline of a yarn is given by the spatial positions of the centres of the yarn cross-sections $\mathrm{O}: \boldsymbol{r}(s)$, where $s$ is coordinate along the midline, $\boldsymbol{r}$ is the radius-vector of the point $\mathrm{O}$. Let $\boldsymbol{t}(s)$ be the tangent to the midline at the point $\mathrm{O}$. The cross-section of the yarn, normal

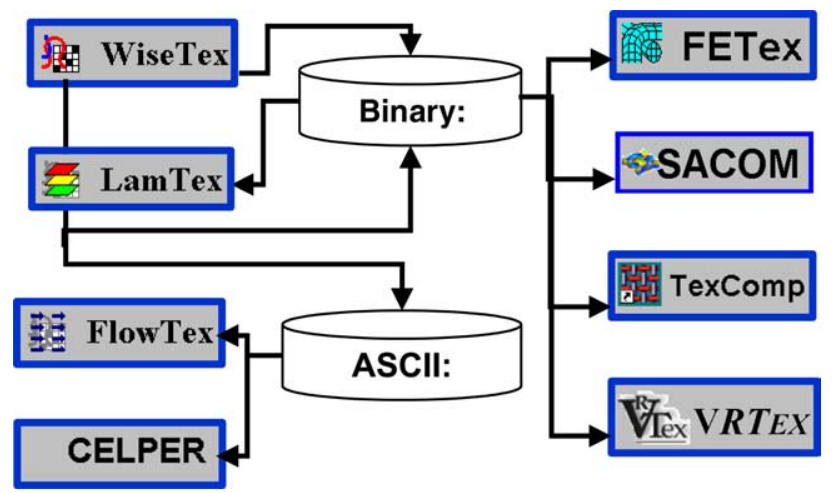

Fig. 2. Data transfer in the WiseTex family of software. to $\boldsymbol{t}$, is defined by its dimensions $d_{1}(s)$ and $d_{2}(s)$ along axis $\boldsymbol{a}_{\mathbf{1}}(s)$ and $\boldsymbol{a}_{\mathbf{2}}(s)$. These axes are "glued" with the cross-section and rotate around $\boldsymbol{t}(s)$, if the yarn is twisted along its path (such a twist can be the result of the fabric shearing [18]). Because of this rotation the sys-

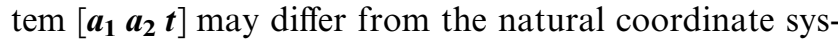
tem along the spatial path $[\boldsymbol{n} \boldsymbol{b} \boldsymbol{t}]$.

The shape of the cross-section can be assumed elliptical, lenticular, etc. The shape type does not change along the yarn, but dimensions $d_{1}$ and $d_{2}$ can change because of different compression of the yarn in the contact zones and between them. Definition of the spatial positions of a yarn with a given cross-section shape in a unit cell consists therefore of five periodic functions: $\boldsymbol{r}(s)$ (then $[\boldsymbol{n b t}]$ vectors can be calculated), $\boldsymbol{a}_{1}(s), \boldsymbol{a}_{2}(s)$, $d_{1}(s), d_{2}(s)$. These functions are calculated for all the yarns in the unit cell by a geometrical model. When used in numerical calculations, all these functions are given as arrays of values for a set of points along the yarn midline.

This description fully defines the volumes of the yarns in a unit cell. The format is the same for orthogonal and non-orthogonal (angle $\alpha$, Fig. 3) unit cells. The in-plane dimensions of the unit cell $X, Y$ are given by the repeat size of the textile structure, whereas the thickness $Z$ is calculated as the difference between the maximum and minimum $z$-coordinates of the cross-sections of all the yarns in the unit cell.

To describe the fibrous structure of the unit cell, consider a point $\mathrm{P}$ and fibrous assembly in the vicinity of this point (Fig. 3). The fibrous assembly can be characterised by physical and mechanical parameters of the fibres near the point (which are not necessarily the same in all points of the fabric), fibre volume fraction $V_{\mathrm{f}}$ and direction $\boldsymbol{f}$ of them. If the point does not lie inside a yarn, then $V_{\mathrm{f}}=0$ and $\boldsymbol{f}$ is not defined. For a point inside a yarn, the fibrous properties are easily calculated,
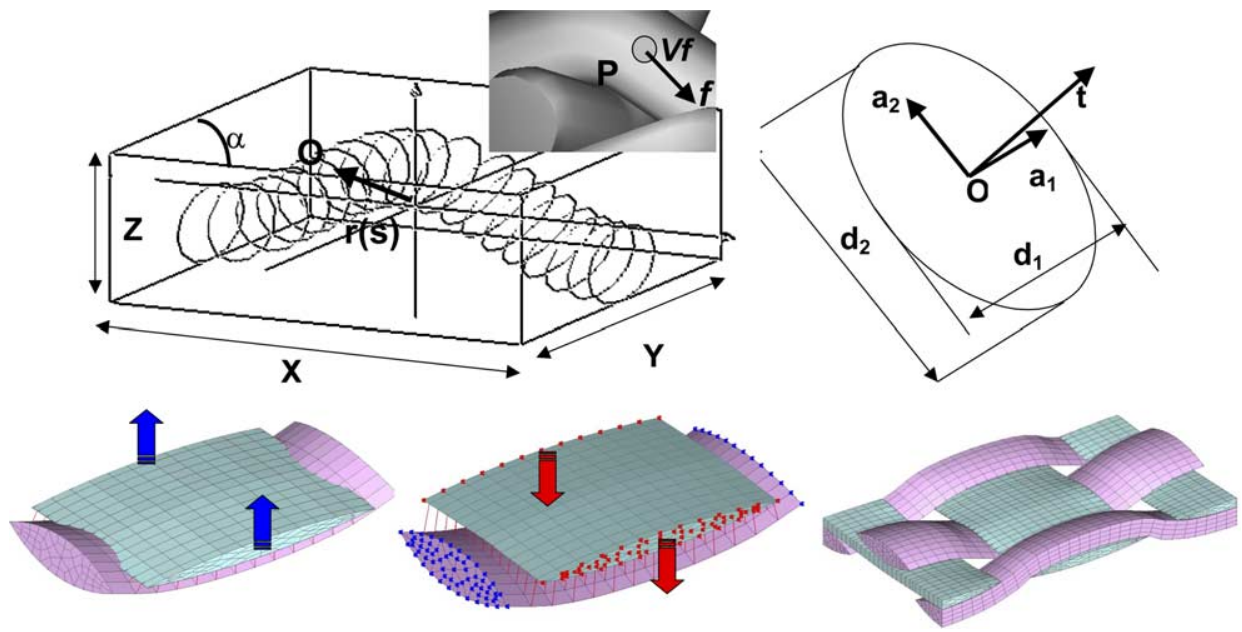

Fig. 3. Set of cross-sections defining a yarn in a unit cell, properties of fibres near point P; meshing of the yarns (developed by Zako et al. [29,36]): two elements with penetrating mesh; separation and "compression"; mesh of the unit cell, no penetration. 
providing that the fibrous structure of the yarns in the virgin state and its dependency of local compression, bending and twisting of the yarn are given. Consider a point $\mathrm{P}$. Searching the cross-sections of the yarns, the cross-sections $S_{i}$ and $S_{i+1}$, containing between their planes the point $\mathrm{P}$, are found (binary search in the unit cell volume is employed to speed up the calculations), and then by interpolation, the cross-section $S$, which plane contains the point $\mathrm{P}$, is built. Using the dimensions of the cross-section $S$, for a given shape of it, point $\mathrm{P}$ is identified as lying inside or outside the yarn. In the former case, with the position of the point $\mathrm{P}$ inside the yarn known, using the model of the yarn microstructure, the parameters of fibrous assembly in the vicinity of the point $\mathrm{P}$ are calculated.

\subsubsection{Translation to finite element model: Woven reinforcements}

The geometry of the yarn volumes can be easily transferred to a finite element description. A section of a yarn between neighbouring cross-sections is subdivided into four volumes (or kept intact if $d_{2} / d_{1}>5$ ). An assembly of these volumes constitutes a solid model of the fabric. For all the yarns a transversal isotropic material model is used, the properties of the impregnated yarns calculated using local fibre volume fraction and fibre orientation in the yarns (assumed constant within one segment between two neighboring cross-sections).

The yarn volumes are effectively meshed, using a "sweep" meshing operation: a mesh built on the first cross-section of the yarn is being "swept" through the yarn, creating a quite regular mesh. Building a mesh inside the matrix is a more challenging proposition. The meshes in the yarns can penetrate one another due to approximations in the geometrical model (Fig. 3). To eliminate the penetration, yarn elements are first separated, with a resistant media placed between them, and then compressed together, so that the edges get to the initial position. The resulting mesh has no penetrations $[29,30]$.

\subsection{Fibrous plies}

\subsubsection{Description of the plies geometry in the textile modelling software}

The description of the geometry of multi-axial multiply stitched preforms (also called "non-crimp fabrics", $\mathrm{NCF}$ ) includes the geometry of the stitching yarns and geometry of the fibrous plies [7].

The stitching, even thin, interacts with the fibrous plies, which are unidirectional arrays of fibres with approximately constant thickness. The stitching causes deviations of the fibres in a ply from their uniform directions. These deviations produce fibre-free zones near stitching locations, which are regularly spaced over the ply. The fibre-free zones can be local (and called "openings" below), or can form continuous channels in the ply (Fig. 4). The local fibre volume fraction in a ply is increased and voids are created as a result of the stitching.

The width and length of the "cracks" and "channels" should be measured or calculated with empirical formulae based on dimensions of the stitching yarn $[20,32]$. When sheared, the fibre density in the plies increases, and the width of the "cracks" and "channels" decreases. After shear angle of $30^{\circ}$, the width reaches its minimum. This change of the width in shear is described by empirical formulae $[32,33]$. The complex geometry of a fibrous ply with "openings" and "channels" is represented in
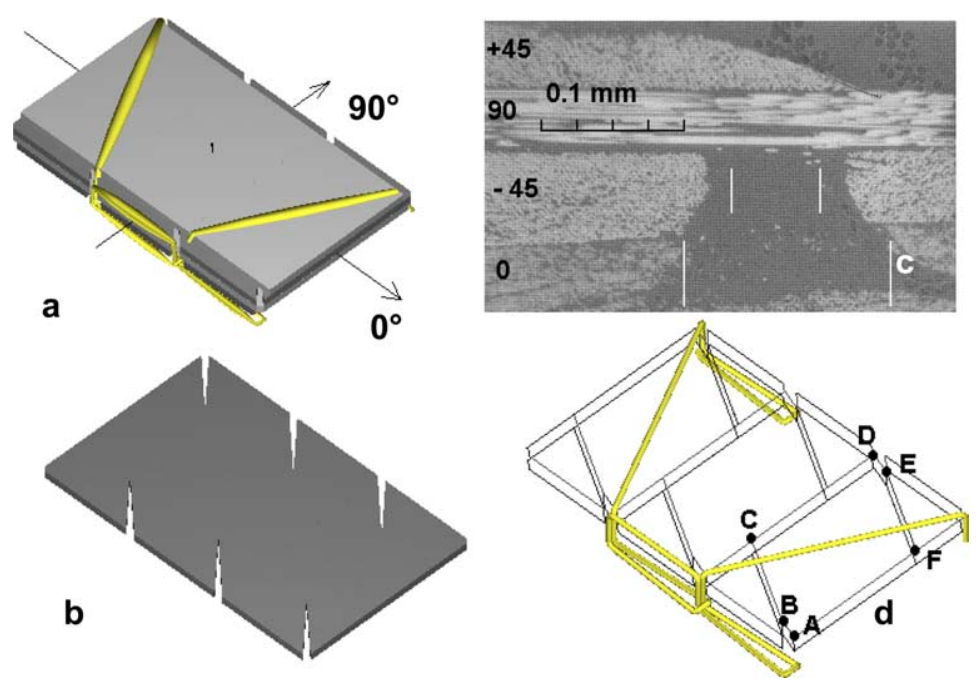

Fig. 4. Quadriaxial NCF, orientation of the fibres in the plies $0 \%-45^{\circ} / 90^{\circ} / 45^{\circ}$. (a) Full geometrical model with stitching. Note a "channel" in the first $0^{\circ}$ ply; (b) "cracks" in the $-45^{\circ} \mathrm{ply}$; (c) Channels/cracks width in cross-sections of the composite plate, thick white lines show width of the cracks as computed by the model, section in the cross-direction, channel in the $0^{\circ}$ face ply and a crack in the $-45^{\circ}$ inner ply; (d) Slab representation of a ply with $-45^{\circ}$ "cracks". ABCDEF - vertices of the upper polygon of one of the slabs. 
the WiseTex model as a set of "slabs". A slab is a volume formed by two parallel polygons. Vertices of all the slabs are stored in the counter-clockwise order (Fig. 4(d)).

\subsubsection{Translation to finite element model: non-crimp fabrics}

The translation to a FE model is done in the following steps [28]: an automatic procedure is used to generate a continuous model satisfying the compatibility conditions. Then, the resulting meshes on the boundary surfaces are built as to have matching node patterns in order for periodic boundary conditions to be applied in the analysis.

Surfaces generated at the geometry decomposition stage are meshed using quadrilateral elements, and the resulting mesh is projected (swept) through the thickness direction, thus obtaining regular hexahedral elements (Fig. 5(a)). Meshes obtained in this way are, in general, unconnected finite element domains whose nodes do not coincide along their common boundaries. In order to preserve nodal connectivity across the interface, a transition region is built by using an intermediate space to glue the partitioned domains using tetrahedral elements (Fig. 5(b)). This technique provides a useful method to satisfy the continuity and the compatibility conditions on non-conforming interfaces between consecutive plies without increasing significantly the computational cost. Finally, matching node patterns are defined on the model boundaries by translating node positions along the fabric periodicity vectors. The FE model obtained in this way is suitable for applying periodic boundary conditions.

\section{Permeability}

Calculation of permeability is based on a voxel representation of the unit cell volume (Fig. 6(a)). A voxel is either empty (pore) or filled with fibres. The flow of the fluid in the pores is governed by Navier-Stokes equations (NS-voxels), inside the permeable tows - by Brinkmann equation (B-voxels). In the latter case, local permeability (micro-level) is calculated with the formulae of Gebart and Berdichevsky for a unidirectional array of fibres. These equations are solved by numerical schemes based on lattice Boltzmann (FlowTex [22]), finite difference (FlowTex, currently being developed sing the solver based on the algorithms of Griebel et al. [34]) or finite element algorithms (CELPER [23]). The homogenised permeability of a unit cell is then determined using an average flux of the fluid through the unit cell under periodic boundary conditions for the given pressure difference on the unit cell facets.

Fig. 6(b) illustrates the calculation of flow through a fabric made of monofilament fibres. The precise definition of the geometry, available for this type of fabric, results in a very good prediction of the permeability: measured [35] $270 \pm 20 \mu^{2}$, calculated $330 \mu \mathrm{m}^{2}$ by the lattice Boltzmann method and $270 \mu \mathrm{m}^{2}$ by the finite difference method by FlowTex. When reinforcement with permeable tows is considered, the correct calculation
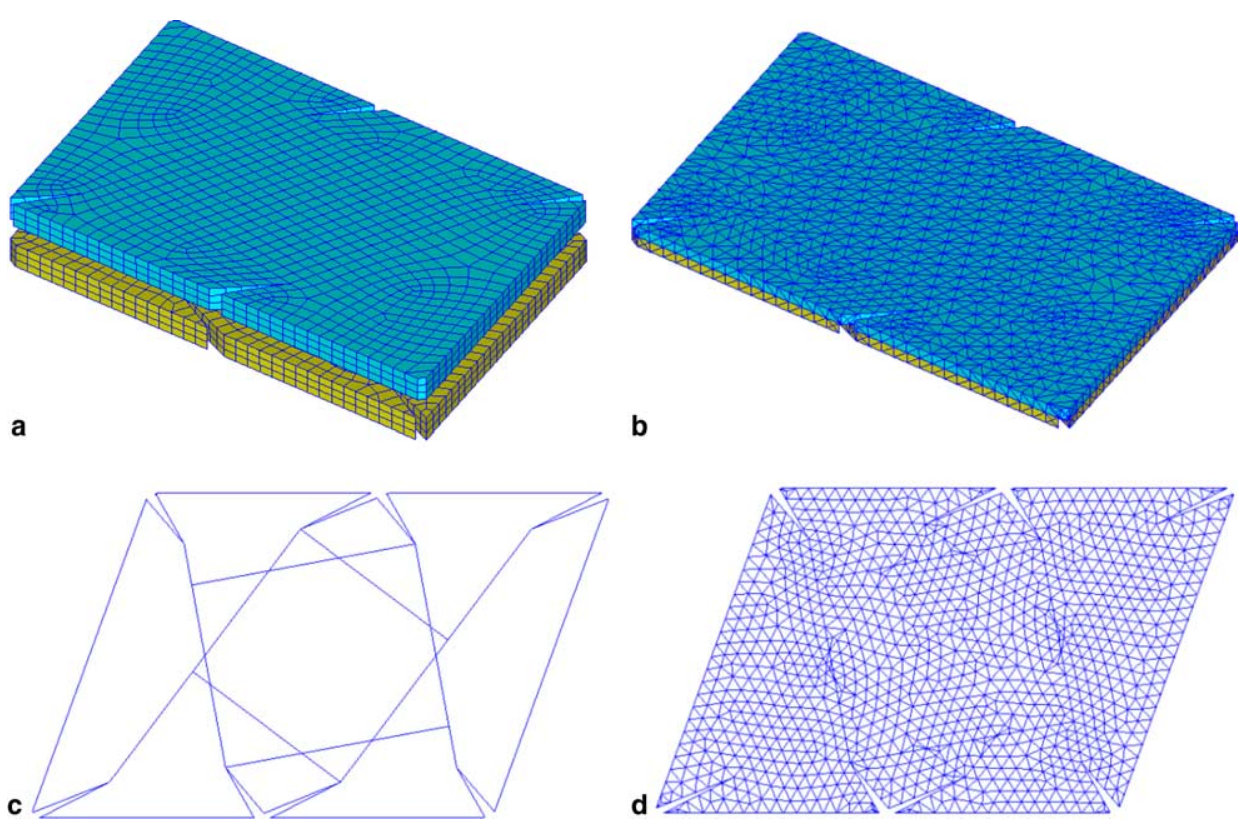

Fig. 5. Automatic meshing process for NCF. (a) Hexahedral finite elements created by sweeping operation; (b) tetrahedral mesh built in the transition region; (c) composite surface at the interface between plies; (d) 2D mesh at the interface. 

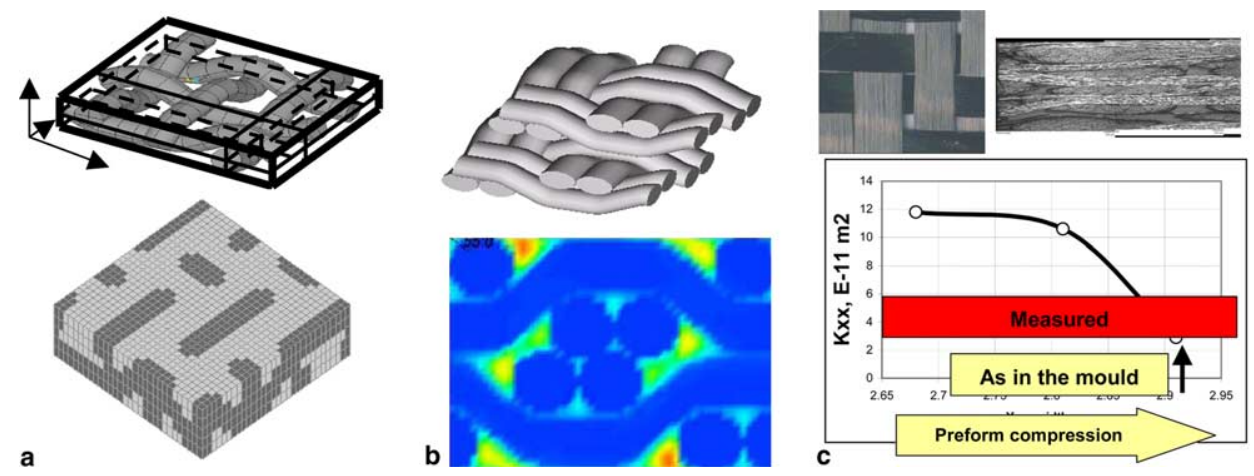

Fig. 6. Calculation of permeability: (a) unit cell and voxel model; (b) two layers of monofilament fabric: WiseTex/LamTex model and flow velocity field (FlowTex); (c) carbon woven reinforcement: fabric, cross-section of the laminate $\left(V_{\mathrm{f}}=55 \%\right)$, CELPER calculations with different compression of the fabric.

of the preform compression is of major importance. Fig. 6(c) illustrates how CELPER calculations come close to the measurements, when the correct parameters of the reinforcement are chosen.

\section{Mechanical properties and damage behaviour}

\subsection{Method of inclusions}

To apply the method of inclusions, implemented in the TexComp software [12-15], the yarns in the unit cell are subdivided into a number of smaller segments, where each yarn segment is geometrically characterised by its total volume fraction, spatial orientation, crosssectional aspect ratio and local curvature (all these parameters are readily provided by the geometrical model). Next, Eshelby's equivalent inclusion principle is adopted to transform each heterogeneous yarn segment into homogeneity with a fictitious transformation strain distribution. The solution makes use of a short fibre equivalent, which physically reflects the drop in the axial load carrying capability of a curved yarn with respect to an initially straight yarn. Every yarn segment is hence linked to an equivalent short fibre, possessing an identical cross-sectional shape, volume fraction and orientation as the original segment it is derived from. The length of the equivalent fibre on the other hand is related to the curvature of the original yarn. For textiles with smoothly varying curvature radii, a proportional relationship between the short fibre length and the local yarn curvature radius is the most straightforward choice and sufficiently accurate for the present purpose.

The interaction problem between the different reinforcing yarns is solved in the traditional way, by averaging out the image stress sampling over the different phases. If a Mori-Tanaka scheme is used, the stiffness tensor $\mathbf{C}^{\mathrm{C}}$ of the composite is hence obtained as: $\mathbf{C}^{\mathrm{C}}=\left[c_{\mathrm{m}} \mathbf{C}^{\mathrm{m}}+\left\langle c_{\mathrm{s}} \mathbf{C}^{\mathrm{s}} \mathbf{A}^{\mathrm{s}}\right\rangle\right]\left[c_{\mathrm{m}} \mathbf{I}+\left\langle c_{\mathrm{s}} \mathbf{A}^{\mathrm{s}}\right\rangle\right]^{-1}$, where the subscripts $\mathrm{m}$ and $\mathrm{s}$ denote the matrix and a yarn segment respectively, $c_{\mathrm{i}}$ is the volume fraction of phase $\mathrm{i}$ $(=\mathrm{m}, \mathrm{s})$, and the angle brackets denote a configurational average.

As follows from this brief description, the homogenisation procedure does not depend on the configuration of the unit cell. Fig. 7 illustrates the homogenisation results for a unit cell of a twill fabric glass composite for different shear angles. Such calculations are used (see below) for multi-level $\mu-\mathrm{m}-\mathrm{M}$ modelling of composite parts.
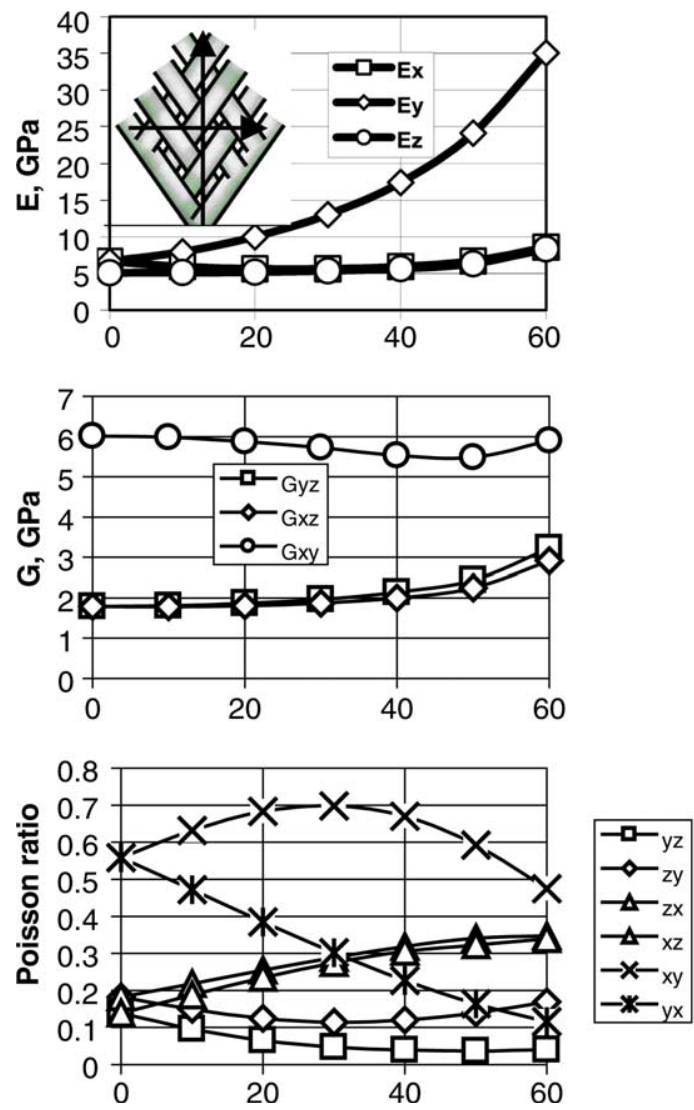

Fig. 7. Elastic properties of a sheared unit cell of a twill glass fabric as functions of shear angle. 


\subsection{FE modelling of elastic and damage behaviour}

The meshing procedure for woven fabrics is implemented in the software MeshTex, which is integrated with WiseTex, forming a geometrical and meshing preprocessor for a finite element solver SACOM $[29,30,36]$. The data flow and FE modelling of 3Dwoven glass/epoxy composites are illustrated in Fig. 8. The 'convertor' within MeshTex converts the WiseTex output into a FE-model, also taking into account the boundary conditions and loads imposed by the problem to be solved. The SACOM-software then calculates local stresses and strains, and by averaging them, issues also the initial stiffness tensor (before damage occurs).

Fig. 9 compares results of the FE modelling of elastic deformation of woven cabon/epoxy composite with full field optical measurements [37]. To enable a clear comparison between the calculated and the measured strain fields, the strains along a line in the $x$ - and another line in the $y$-direction are shown. It can be observed that there is a good match between both; moreover, a strong variation of the strains depending on the location relative to the warp and weft yarns indicates that reliable local strain analyses are essential to predict damage development in textile based composites.
The FEA of progressive damage of the stitched NCF composite was performed by Carvelli et al., assuming the Tsai-Hill failure criteria for the anisotropic homogenized layers. The Tsai-Hill strength parameters of the homogenized layers are derived from a micro model, assuming a transversally isotropic carbon fibres and an isotropic matrix. A simple degradation procedure, proposed by Zako [38-40], is applied to evaluate the damage in the layers up to complete failure. When the stress state in an element satisfies the assumed failure criterion, certain (depending of the failure mode) stiffness matrix elements are dropped down.

As shown in Fig. 10, the FE-analysis accounts for the presence of resin-rich zones (channels), created by the stitching. It gives good estimation of the initial damage strain (red arrows, at about $0.3 \%$ strain in both graphs), which was also measured $[41,42]$ using $\mathrm{AE}$ registration and X-ray observations of damage.

\section{Micro-meso-macro structural analysis}

Fast homogenisation with the inclusion model is wellsuited to be used in micro-macro calculations. Simulation of the performance of textile composite parts takes

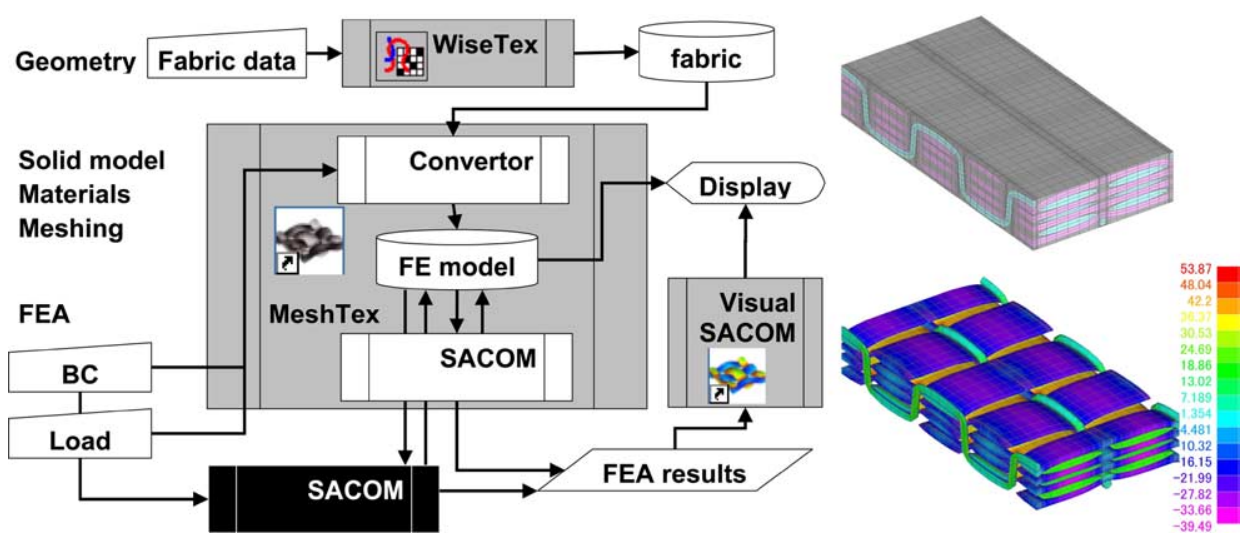

Fig. 8. WiseTex-MeshTex-SACOMdata flow and example of FEA of 3D-woven composite.
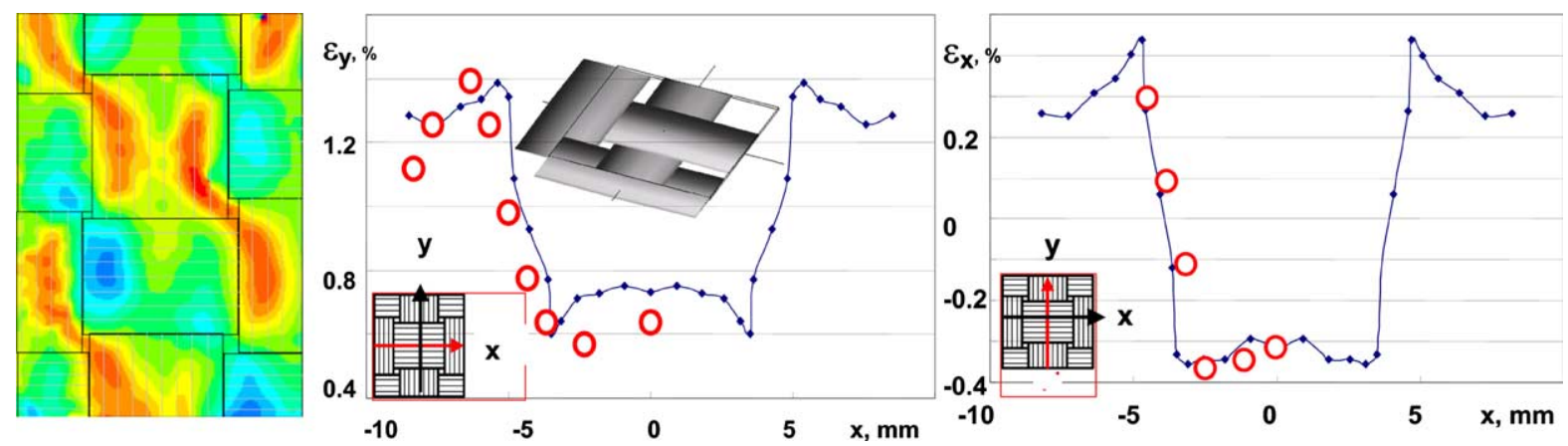

Fig. 9. Full field strain registration [37] on the surface of carbon-epoxy (one fabric layer) and FEA: longitudinal and transversal strain along the yarn centre line (points - experiment, lines - FEA). 

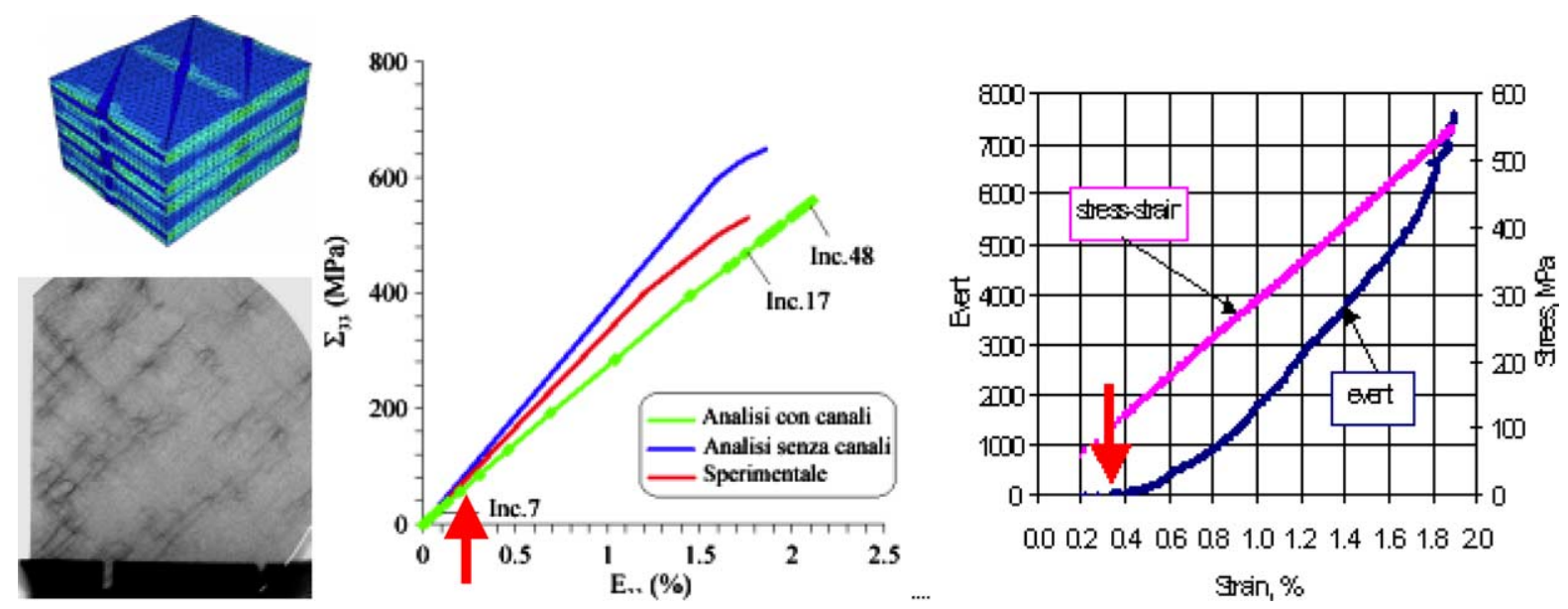

Fig. 10. FEA of damage in NCF carbon/epoxy composites: FE model, X-ray registration of damage, at a strain of $0.8 \%$ : calculated and measured stress-strain diagrams with ("con") and without ("senza") resin-rich zone ("canali") produced by the stitching; AE registration of damage. Arrows show initial damage strain $(0.3 \%)$ in calculation and experiment.

into account variability of local properties of the composite, as a 3D-shaped textile preform undergoes shear deformations in the processing. The intensity of shear varies from point to point, changing the local properties of the composite part.

As reported in detail in [24,25], four different parts (Fig. 11(a)) were modelled with commercial CAD software packages providing a geometrical description, which was transferred to a FE mesh that is needed for further simulations (graphs below show results for woven glass/epoxy hemisphere with diameter of $400 \mathrm{~mm}$ ).

Although a kinematical draping algorithm (QuikForm) is used and hence the material properties are not taken into account during the draping simulation, the prediction of the textile deformation is relatively accurate (Fig. 11(b)). The local stiffness of the parts was calculated as follows. The QuikForm output file contains shear angles of the reinforcement in all the
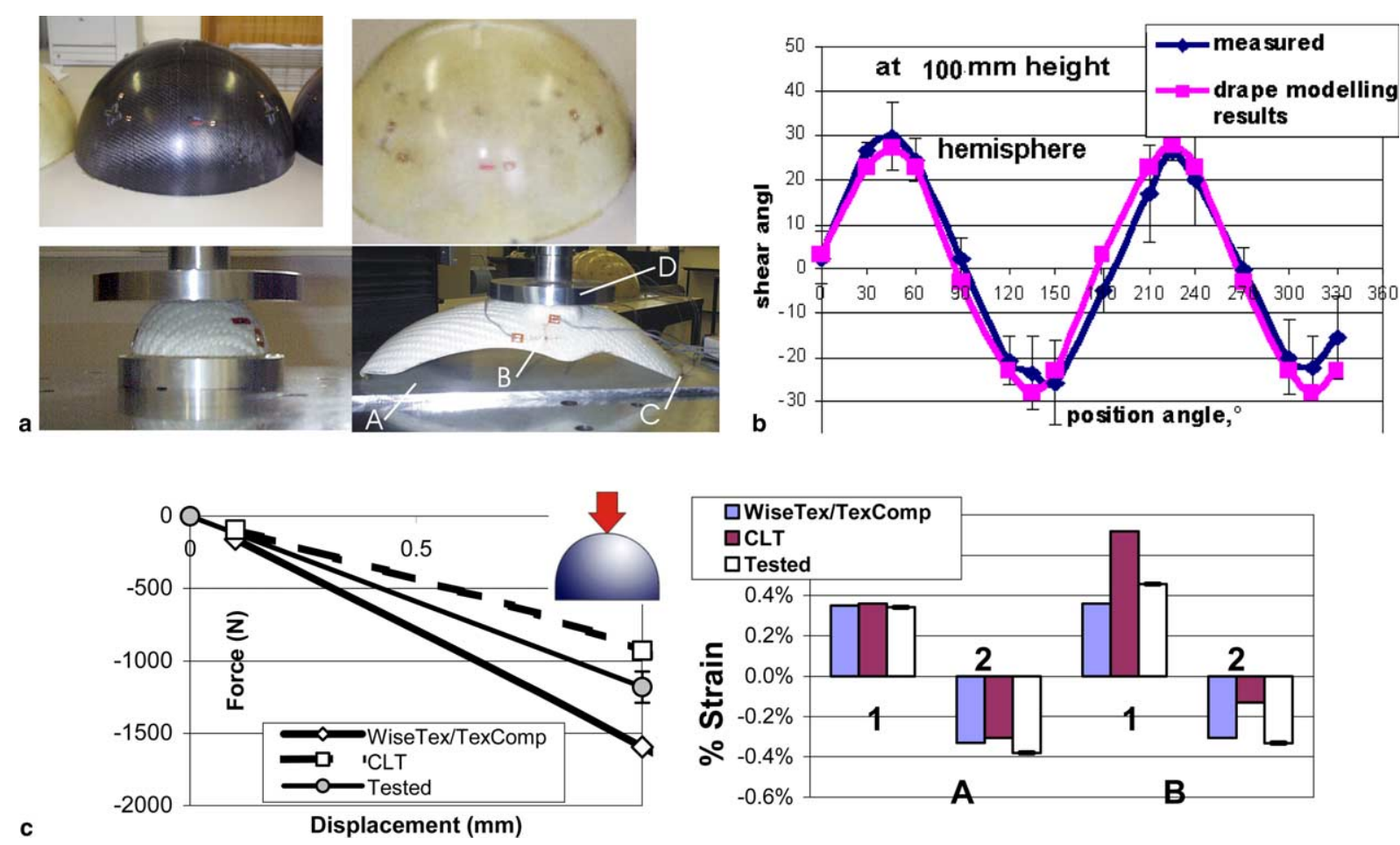

Fig. 11. Micro-macro analysis: (a) composite parts: NCF carbon/epoxy and woven glass/epoxy hemispheres, Twintex glass/PP hemisphere, Twintex motorbike mudgard; (b) draping: simulation and measurements, glass/epoxy hemisphere.; (c) experimental and calculated load-deflection curves (left) and local strains (right, 1-mm displacement of the compression head). Glass/epoxy hemisphere, (1) strain in horizontal direction; (2) strain in vertical direction. (A) low shear positions; (B) high shear positions. 

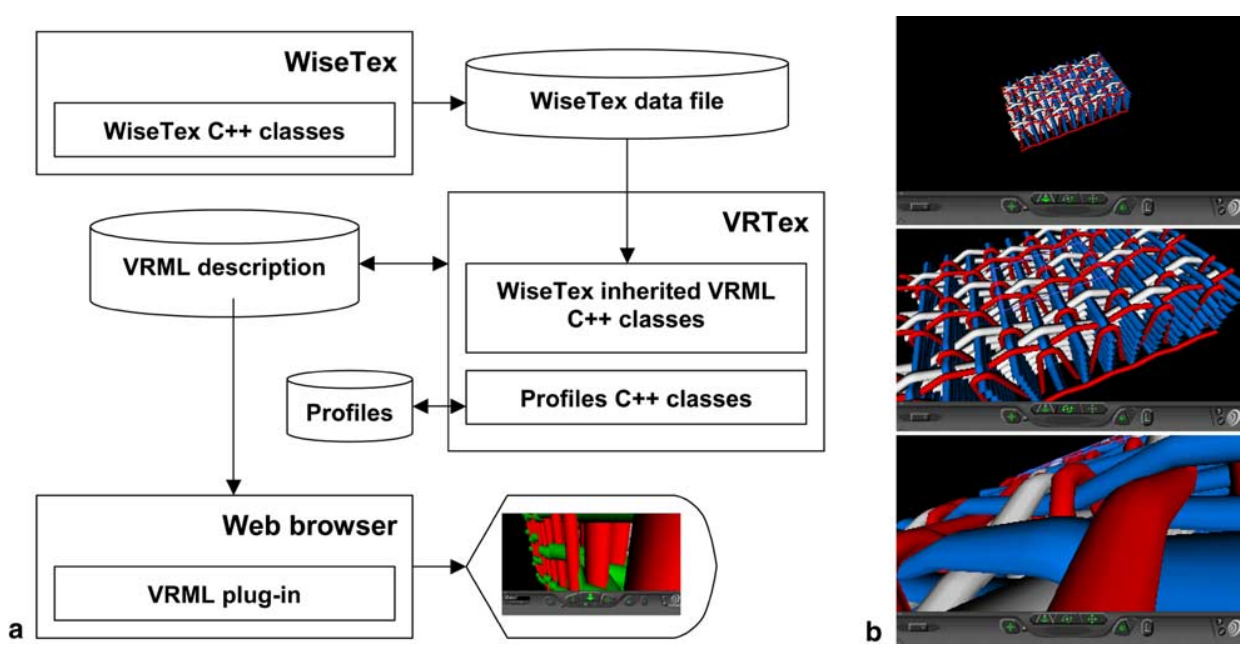

Fig. 12. VRTex: data transfer scheme (a) and travel in textile virtual world (b).

elements of the mesh (the difference between shear of the different layers of the preform was neglected). For each element WiseTex calculated the internal geometry of the sheared reinforcement. The result was processed by TexComp to produce the full stiffness matrix of the impregnated reinforcement in the element. This calculation accounts for the differences of the properties in elements induced by local shear: (1) fibre volume fraction; (2) in-plane orientations of the yarns and fibres inside them; (3) out-of-plane orientations of the yarns in the sheared woven structure.

The parts were tested in compression at the constant displacement rate of $1 \mathrm{~mm} / \mathrm{min}$. Care was taken to ensure that the boundary conditions correspond to rigid clamping of the part edges: they were glued to the base with epoxy glue. Local strains on the surface of the parts were registered using strain gages. A FE-analysis ( $S Y S$ $P L Y)$ was performed using stiffness of the elements calculated with: (1) WiseTex/TexComp; (2) simple model based on Classical Laminate Theory and in-plane changes of the fibre orientations.

Comparison (Fig. 11(c)) between the global part behaviour (force-displacement for the whole part) during loading and the FE simulation shows a reasonable prediction of the part stiffness in the elastic region. For local strains, the 'micro-macro modelling' technique based on the homogenisation of a real reinforcement textile structure approaches the tested values better than a simplified model, especially in areas where textile structures are highly deformed during production (large shearing angle of the fabric), as it could be expected.

\section{Textile virtual reality}

One of important applications of models of internal geometry is visualisation of the textile architecture. The ultimate result of visualisation of a model is the creation of a virtual reality (VR) world. Up to now, complicated textile VR is being created mostly on macro-level, displaying drape of a cloth and serving needs of garment designers and producers of animations, supported by the appeal for the wide public. Micro-level (internal structure) textile VR (below we will also use a shortened term "micro-VR") attracts lesser attention. However, such an application is of great interest for textile education and for the popularisation of textile industry and science (e.g., interactive exhibits for industrial and science museums). It can be also used for research purposes in the same way as stereo-chemistry takes advantage of 3D-models of large molecules.

The VRTex software is a tool (developed by Mikolanda [31,43]), which transforms a WiseTex data file to the virtual reality data files according to VRML standard, which can be used for any VRML browser, providing $3 \mathrm{D}$ visualisation, navigation and interaction facilities. VRML commands, defining the VR-world, are generated by VRTex for all the yarns and the fibrous plies in the fabric unit cell. These definitions are translated in three directions as requested by the user; VRML instruments allow doing this almost without penalty in the file size, but the user interaction time increases linearly with the enlargement of the virtual world. Fig. 12 shows the usage of VRTex, illustrating "travelling" in the virtual world.

\section{Conclusion}

The WiseTex family of models and software provides an integrated description of the internal geometry and properties of textile fabrics and composites on unit cell level and is used for multilevel $\mu-\mathrm{m}-\mathrm{M}$ analysis. The general format of description of the internal geometry of textile reinforcements is open to be used in further developments. 


\section{Acknowledgements}

The work reported here was supported by EC (TECABS, ITOOL and Marie Curie projects), Government of Flanders (STWW and GBOU projects) and Research Council of K.U.Leuven (GOA and OT projects). The contributions of all the members of the international team, mentioned earlier, are greatly acknowledged as well.

\section{References}

[1] Vandeurzen P. Structure-performance modelling of two-dimensional woven fabric composites. Leuven: Department of MTM, Katholieke Universiteit Leuven; 1998.

[2] Vandeurzen P, Ivens J, Verpoest I. Micro-stress analysis of woven fabric composites by multilevel decomposition. J Compos Mater 1998;32(7):623-51.

[3] Gommers B, Verpoest I, Van Houtte P. The Mori-Tanaka method applied to textile composite materials. Acta Mater 1998;46(6):2223-35.

[4] Prodromou A. Mechanical modelling of textile composites utilising a cell method. Leuven: Department of MTM, Katholieke Universiteit Leuven; 2004. p. 149.

[5] Lomov SV, Truevtzev NN. A software package for the prediction of woven fabrics geometrical and mechanical properties. Fibres Text East Eur 1995;3(2):49-52.

[6] Shtut II, Bezin PB, Lomov SV. Optimizing the fibrous content and properties of blended SVM-cotton yarns. Chimicheskie Volokna 1995(4):31-3.

[7] Lomov SV, Gusakov AV. Modellirung von drei-dimensionalen gewebe Strukturen. Tech Text 1995;38:20-1.

[8] Lomov SV, Primachenko BM, Truevtzev NN. Two-component multilayered woven fabrics: weaves, properties and computer simulation. Int J Cloth Sci Technol 1997;9:98-112.

[9] Gusakov AV, Lomov SV. Parametric studies of the internal structure of 3D-woven fabrics. Fibres Text East Eur 1998;6(2):60-3.

[10] Lomov SV, Gusakov AV. Computation of the porosity of one and multi-layered woven synthetic fabrics. Chimicheskie Volokna 1998:52-5.

[11] Lomov SV, Gusakov AV. Mathematical modelling of 3D and conventional woven fabrics. Int $\mathrm{J}$ Cloth Sci Technol 1998;10(6):90-1.

[12] Huysmans G, Verpoest I, Van Houtte P. A poly-inclusion approach for the elastic modelling of knitted fabric composites. Acta Mater 1998;46(9):3003-13.

[13] Huysmans G, Verpoest I, Van Houtte P. A damage model for knitted fabric composites. Compos Part A 2001;32(10): $1465-75$.

[14] Lomov SV, Huysmans G, Luo Y, Parnas R, Prodromou A, Verpoest I, et al. Textile composites models: integrating strategies. Compos Part A 2001;32(10):1379-94.

[15] Lomov SV, Gusakov AV, Huysmans G, Prodromou A, Verpoest I. Textile geometry preprocessor for meso-mechanical models of woven composites. Compos Sci Technol 2000;60:2083-95.

[16] Lomov SV, Huysmans G, Verpoest I. Hierarchy of textile structures and architecture of fabric geometric models. Text Res J 2001;71(6):534-43.

[17] Lomov SV, Truong Chi T, Verpoest I, Peeters T, Roose V, Boisse P. Mathematical modelling of internal geometry and deformability of woven preforms. Int J Form Process 2003; 6(3-4):413-42.
[18] Lomov SV, Nakai A, Parnas RS, Bandyopadhyay Ghosh S, Verpoest I. Experimental and theoretical characterisation of the geometry of flat two- and three-axial braids. Text Res J 2002;72(8):706-12.

[19] Moesen M, Lomov SV, Verpoest I. Modelling of the geometry of weft-knit fabrics. In: TechTextil symposium, Frankfurt; 2003 [CD edition].

[20] Lomov SV, Belov EB, Bischoff T, Ghosh SB, Truong Chi T, Verpoest I. Carbon composites based on multiaxial multiply stitched preforms. Part 1: Geometry of the preform. Compos Part A 2002;33(9):1171-83.

[21] Lomov SV, Verpoest I, Peeters T, Roose D, Zako M. Nesting in textile laminates: geometrical modelling of the laminate. Compos Sci Technol 2002;63(7):993-1007.

[22] Belov EB, Lomov SV, Verpoest I, Peeters T, Roose D, Parnas RS. Modelling of permeability of textile reinforcements: lattice Boltzmann method. Compos Sci Technol 2004;64:1069-80.

[23] Laine B, Hivet G, Boisse P, Boust F, Lomov SV. Permeability of the woven fabrics: a parametric study. In: Proceedings ESAFORM-8 conference; 2005. p. 995-8.

[24] Van den Broucke B, Tumer F, Lomov SV, Verpoest I, De Luka P, Dufort L. Micro-macro structural analysis of textile composite parts: case study. In: Proceedings of the 25th international SAMPE Europe conference, Paris; March 30th-April 1st 2004. p. 194-99.

[25] Lomov SV, Van den Broucke B, Tumer F, Verpoest I, De Luka P, Dufort L. Micro-macro structural analysis of textile composite parts. In: Proceedings of ECCM-11, Rodos; 2004 [CD edition].

[26] Carvelli V, Truong Chi T, Larosa M, Lomov SV, Poggi C, Ranz Angulo D, et al. Experimental and numerical determination of the mechanical properties of multi-axial multi-ply composites. In: Proceedings of ECCM-11, Rodos; 2004 [CD edition].

[27] Lomov SV, Verpoest I, Kondratiev SV, Borovkov AI. An integrated model strategy for processing and properties of textile composites: new results. In: McCarthy RFJ, editor. Proceedings of the 22nd international SAMPE Europe conference. Paris: SAMPE; 2001. p. 379-89.

[28] Lomov SV, Bernal E, Ivanov DS, Kondratiev SV, Verpoest I. Homogenisation of a sheared unit cell of textile composites: FEA and approximate inclusion model. Revue européenne des éléments finis [in print].

[29] Lomov SV, Ding X, Hirosawa S, Kondratiev SV, Molimard J, Nakai H, et al. FE simulations of textile composites on unit cell level: validation with full-field strain measurements: In: Proceedings of the 26th SAMPE-Europe conference, Paris; 5th-7th April 2005 [CD edition].

[30] Kurashiki T, Zako M, Hirosawa S, Lomov SV, Verpoest I. Estimation of a mechanical characterization for woven fabric composites by FEM based on damage mechanics. In: Proceedings of ECCM-11, Rodos; 2004 [CD edition].

[31] Lomov SV, Mikolanda T. Textile virtual reality. In: Proceedings of TechTextil symposium, Frankfurt am Main; 2005 [CD edition].

[32] Loendersloot R, Lomov SV, Akkerman R, Verpoest I. Carbon composites based on multiaxial multiply stitched preforms. Part 5: Geometry of sheared biaxial fabrics. Compos Part A [in print].

[33] Loendersloot R, Lomov SV, Akkerman R, Verpoest I. Architecture and permeability of sheared carbon fibre non-crimp fabrics. In: Drechsler K, editor. Proceedings of the 24th international SAMPE Europe conference, Paris; 2003. p. 141-8.

[34] Griebel M, Dornseifer T, Neunhoeffer T. Numerical simulation in fluid dynamics, a practical introduction. Philadelphia: SIAM; 1998.

[35] Hoes K, Sol H, Dinescu D, Lomov SV, Parnas R. Study of nesting induced scatter of permeability values in layered reinforcement fabrics. Compos Part A 2004;35(12):1407-18.

[36] Kurashiki T, Hirosawa S, Zako M, Lomov SV, Verpoest I. On a numerical simulation of the mechanical behaviour for laminated 
woven fabric composites under tensile loading. In: Proceedings of the 7th international conference on textile composites (TexComp7), Yonezawa; 2004. p. 1-4 [Textile 13].

[37] Lee JR, Molimard J, Vautrin A, Surrel Y. Digital phase-shifting grating shearography for experimental analysis of fabric composites under tension. Compos Part A 2004;35:849-59.

[38] Kurashiki T, Zako M, Verpoest I. Damage development of woven fabric composites considering an effect of mismatch of lay-up. In: Degrieck J, editor. Composites for the future, proceedings of the 10th European conference on composite materials (ECCM-10), Brugge; 2002 [CD edition].

[39] Uetsuji Y, Zako M, Nishiyabu K. Numerical analysis and in situ SEM observation of damage development for woven fabric composite materials. J Soc Mater Sci Jpn 2002;51(10): $1147-53$.
[40] Zako M, Uetsuji Y, Kurashiki T. Finite element analysis of damaged woven fabric composite materials. Compos Sci Technol 2003;63:507-16.

[41] Truong Chi T, Vettori M, Lomov SV, Verpoest I. Carbon composites based on multiaxial multiply stitched preforms. Part 4: Mechanical properties of composites and damage observation. Compos Part A [in print].

[42] Vettori M, Truong Chi T, Lomov SV, Verpoest I. Progressive damage characterization of stitched, biaxial, multi-ply carbon fabrics composites. In: Proceedings of ECCM-11, Rodos; 2004 [CD edition].

[43] Mikolanda T, Lomov SV, Kosek M, Verpoest I. Simple use of virtual reality for effective visualization of textile material structures. In: CODATA Prague workshop on information visualization, presentation, and design, Prague; 2004 [CD edition]. 